\title{
Dilemas e perspectivas da abordagem sociológica dos desastres naturais
}

Marcos Mattedi

\section{A problemática sociotécnica dos desastres naturais}

Os desastres naturais constituem um tema de estudo muito controverso. Após quase um século de pesquisa sistemática sobre o tema, na sociologia não existe ainda consenso sobre o que é um desastre e sua definição encontra-se em negociação (Quarantelli, 1998; Quarantelli e Perry, 2005). Essa disputa reflete tanto a diversidade de disciplinas mobilizadas no debate, por exemplo, a sociologia, a geografia, a antropologia, a epidemiologia, a psicologia e as disciplinas técnicas, meteorologia, sismologia, engenharias etc. (Alexander, 2000), como também as ambivalências dentro de uma mesma disciplina, como é o caso da sociologia (Tierney, 2007). Além disso, essas disputas refletem as estratégias de gestão e resposta (Revet, 2009). Nesse sentido, a temática dos desastres constitui um espaço de tensão porque abriga interesses muito heterogêneos. A definição dos desastres constitui, portanto, um desafio ao mesmo tempo cognitivo e político.

Esses impasses conceituais parecem refletir o contexto sociocognitivo de desenvolvimento institucional da pesquisa dos desastres. A preocupação com os desastres possui uma longa história (Bryant, 1991; Schenk, 2007; Kozák e Cermák, 2010) e atenção da mídia (Pantti et al., 2012) e estes são onipresentes na vida cotidiana das pessoas (Crowe, 2012). As primeiras contribuições remontam ao início do século (Prince, 1920; Carr, 1932), mas é somente após a Segunda Guerra Mundial que se estabelece um processo de produção sistemática de informações (Quarantelli, 1978). 
Com o tempo, a produção do conhecimento sobre desastres se institucionalizou, penetrando na agenda governamental e disseminando-se globalmente. Hoje constitui uma área de estudos que possui vários grupos de pesquisa, revistas especializadas, comitês em associações científicas, uma instituição vinculada à ONU e também um conjunto de iniciativas de redução dos riscos de desastres. Isso indica que quanto mais a problemática dos desastres se dissemina, mais controversa se torna sua abordagem.

A preocupação sociológica com os desastres naturais encerra as próprias transformações da percepção moderna da natureza. Isso significa que abordar os desastres naturais pressupõe a consideração dos dispositivos de como a segurança e o risco são selecionados socialmente. Esse processo se manifesta no contínuo desenvolvimento de informações, discursos, ferramentas, normas, crenças, organizações etc. relacionadas, principalmente, com as questões ambientais, tecnológicas, alimentares e sanitárias (Beck, 1992; Luhmann, 1992; Douglas e Wildavsky, 1982). Assim, sociedade e natureza vão deixando de ser consideradas como entidades fixas e a própria separação natural-social se transforma em pontos de negociação sociotécnica (Latour, 1999). Portanto, a modificação das concepções de natureza faz com que, inclusive, eventos que historicamente eram considerados como exteriores à sociedade passem a ser considerados como produzidos socialmente.

Os dados disponíveis sobre a gestão dos desastres naturais também exprimem essas disputas. Por um lado, mostram o fortalecimento da capacidade de monitoramento global e, por outro, o aumento da incerteza aos impactos locais (UNISDR, 2015). Apesar dos esforços nas últimas décadas para deslocar o foco das Respostas a Crises de Emergência (RCE) pós-impacto para a Redução do Risco de Desastres (RRD) pré-impacto, os impactos refletem as desigualdades de desenvolvimento, pois as perdas se concentram nos países e grupos de menor renda (Wisner et al., 1994). A gestão dos desastres foi sendo progressivamente integrada às questões da ajuda humanitária, do desenvolvimento e do meio ambiente mobilizando atores muito heterogêneos. Nesse sentido, o aumento da circulação de informações e a proliferação de discursos sobre a vulnerabilidade aumentou os níveis de tensão sobre a gestão dos desastres (Revet, 2009).

O desenvolvimento da abordagem sociológica dos desastres parece refletir esses impasses cognitivos e políticos. Assim, partindo dos desafios que caracterizaram o processo de incorporação dos desastres pela sociologia (Fritz, 1961), passando pelos exercícios de sínteses teóricas que tentaram estabilizar sua abordagem (Quarantelli e Dynes, 1977; Kreps, 1984; Quarantelli, 1989), até chegar ao processo de desenvolvimento disciplinar recente (Gilbert, 1995; Perry, 1989, Tierney, 2007), o desafio parece sempre o mesmo: definir O que é um desastre? (Quarantelli, 1998; Quarantelli e Perry, 2005). Muito embora esses exercícios teóricos representem necessidades historicamente localizadas de delimitar o campo de estudos dos de- 
sastres na sociologia, as controvérsias relativas à abordagem sociológica parecem se concentrar em torno de três eixos temáticos: (a) agentes do desastre; (b) unidades sociais impactadas; (c) padróes de resposta.

A consideração dessas três unidades de análise permite compreender as dificuldades de estabilização de uma estrutura conceitual na abordagem sociológica dos desastres. Mais precisamente, argumentamos que o desenvolvimento disciplinar da abordagem sociológica da problemática dos desastres encerra três paradoxos:

- Paradoxo da informação: desastres são produzidos socialmente, mas são pouco conhecidos pela sociedade.

- Paradoxo da intensificação: quanto maior o número de pesquisadores, menos produtiva a área de estudo.

- Paradoxo da gestão: quanto mais sofisticados os dispositivos de gestão, mais destrutivos os desastres.

Esses desafios dizem respeito às relações que a ciência estabelece com o contexto social. Para entender esses paradoxos precisamos reorientar as estratégias de abordagem dos desastres e considerar o papel que a ciência e a tecnologia desempenham na organização da sociedade moderna. Mais precisamente, precisamos recorrer a subsídios fornecidos pela abordagem sociológica da ciência e da tecnologia.

A sociologia da ciência ocupa-se da relação que se estabelece entre atividade científica e contexto social. A operacionalização do contexto social permite a diferenciação de três estratégias de abordagem: (1) a sociologia dos cientistas (Merton, 1996); (2) a sociologia do conhecimento cientifico (Bloor, 1991; Collins, 1992); (3) a sociologia da pesquisa cientifica (Latour, 2000; Callon, 1986). Considerando esses subsídios, argumentamos que os padrões cognitivos da sociologia dos desastres devem ser considerados com base nas contribuições da própria sociologia, nas relações que a sociologia mantém com outras disciplinas e com a sociedade. Nesse sentido, (i) na primeira parte trataremos das disputas com outras disciplinas pela caracterização dos desastres; (ii) em seguida da disputa metodológica dentro da própria sociologia pela descrição do impacto; (iii) passando pela disputa com outros peritos pela orientação das políticas de gestão dos desastres; (iv) e para finalizar em forma de síntese avançaremos uma definição de desastre.

A controvérsia interdisciplinar: a oposição social-natural

A controvérsia interdisciplinar está relacionada com a disputa que a sociologia estabelece com outras disciplinas na definição dos desastres. Apesar de não existirem 
critérios formais para a definição de uma disciplina científica (Lemaine et al., 1976), as disciplinas constituem a unidade primária de diferenciação interna do sistema de ciência (Stichweh, 1992). As disciplinas científicas podem ser caracterizadas como organizações sociais especializadas em identificar e resolver problemas dentro de culturas epistêmicas institucionalizadas academicamente (Cetina, 1999). As disciplinas operam por meio de um Modelo Problema-Solução baseado numa forma especializada de produção do conhecimento, dotada de formação universitária, organizações científicas e veículos de comunicação. Essa operação se caracteriza por um processo de diferenciação externa e integração interna. Dentro desse modelo problema-solução, a disputa interdisciplinar se estabelece em torno da caracterização dos agentes dos desastres naturais.

Essa disputa refere-se ao caráter multidimensional dos desastres, pois desastres constituem fenômenos que se estabelecem nos pontos de interseção entre o social e o natural (Mattedi e Butzke, 2001). Assim, os desastres naturais podem ser considerados, ao mesmo tempo, fenômenos naturais e fenômenos sociais. A comunidade de pesquisadores sociais sobre desastres é formada por geógrafos e sociólogos, e os impasses dizem respeito, principalmente, à determinação do quanto os desastres naturais são causados por fatores externos e internos à sociedade. É possível diferenciar duas estratégias teórico-metodológicas (Lepointe, 1991; Gilbert, 1995) predominantes de caracterização dos agentes sociais desastres: (a) a abordagem externalista: que enfatiza os aspectos físicos, concentrando atenção sobre as características do evento-percep̧̧ão; (b) abordagem internalista: que enfatiza os aspectos sociais, destacando as características do impacto-organização.

A abordagem externalista está relacionada com a preocupação da geografia com as características do evento. Esse gênero de abordagem tem uma longa tradição de pesquisa nos estudos sobre ajustamento aos hazards. Esse tipo de pesquisa focaliza os aspectos físicos de um tipo especial de evento ambiental que representa ameaças para os seres humanos. Mais precisamente, trata-se de entender como fatores geofísicos contribuem para criação de ambientes ameaçadores - exposição a e percepção de tais ambientes (Montz e Tobin, 2011) - e de relacionar as características físicas dos desastres com os fatores psicológicos dos indivíduos, visando estabelecer o padrão de ajustamento que os indivíduos mantêm com eventos extremos. Portanto, o foco desse tipo de abordagem considera as variações de ajustamento ao ambiente geofísico, ou seja, como os seres humanos selecionam o curso de ação num ambiente de risco, combinando a análise do sistema de classificação dos eventos com os tipos de respostas.

A preocupação com a relação entre o homem e o meio ambiente está associada ao trabalho pioneiro desenvolvido pelo geógrafo Gilbert White em 1945, intitulado Human adjustment to floods (Kates, 2011). White chamou atenção para o fato de 
que a implantação do Flood Control Act de 1936, estabelecida no contexto político do New Deal, como por exemplo, barragens, diques e mudanças de canal, não diminuía os danos. Por isso assinalou a importância de arranjos alternativos com base no ajustamento humano aos desastres. E em 1956, com o auxílio de fundos públicos estabeleceu um grupo de estudos na Universidade de Chicago para examinar a questão do ajustamento humano ao problema das enchentes. Esses estudos foram progressivamente estendidos a outros eventos (terremotos, tornados, furacões, secas etc.), e culminam com a implantação do Natural Hazard Center, em 1976, na Universidade do Colorado em Boulder.

Os estudos de hazards são divididos de acordo com os fatores deflagradores. Os hazards naturais são diferenciados em função dos fatores deflagradores do ambiente físico, considerando aspectos como, por exemplo, a velocidade, a extensão e a duração de sua eventual destruição, a presença dos impactos secundários, o grau de preparação social e a percepção da população ao risco (Cvetkovich e Earle, 1985). Porém, um acompanhamento empírico indica que os processos meteorológicos, geológicos e hidrológicos possuem pouca similaridade entre si. Por isso, pode-se considerar cada espécie de hazards segundo: (a) mecanismos físicos (magnitude, duração, extensão espacial); (b) distribuição temporal (frequência, sazonalidade, parâmetros diurnos); (c) distribuição espacial (localização geográfica); (d) dinâmica de eclosão (rapidez do início, tempo de preparação e rapidez de término) (Tobin e Montz, 1997).

Para descrever os desastres naturais os estudos de hazards recorrem a dois modelos teóricos. Por um lado, recorrem à ecologia humana para descrever os desastres em termos de eventos extremos que surgem dos padrões de relações entre o homem e a natureza; por outro, às teorias da escolha racional para descrever as percepçôes dos eventos em termos de adaptação humana ao ambiente (Burton et al., 1993). Assim, os eventos geofísicos extremos são o ponto de partida para a análise dos desastres, que considera a percepção que os indivíduos possuem dos eventos e os meios de subsistência e toma como unidades específicas de análise grupos sociais ou comunidades. Dessa forma, esse programa de pesquisa seleciona como ponto de partida o ajuste do homem aos hazards e como o seu entendimento afeta as suas respostas. Portanto, a abordagem externalista dos hazards combina os subsídios da geografia física e da geografia humana, articulando Evento (físico) e Percepção (humana).

A abordagem internalista focaliza os aspectos sociais dos desastres através da relação impacto-organização. As respostas humanas nunca são dependentes somente dos processos geofísicos e não podem ser explicadas do ponto de vista individual. Isso significa que os desastres variam mais socialmente que geofisicamente na medida em que são processos sociais que determinam a intensidade da destruição de um evento natural. Mais precisamente, cada contexto social conecta a rotina cotidiana 
de sobrevivência aos acontecimentos extraordinários de forma específica (Gilbert, 1995). Assim, a gravidade dos desastres naturais quase sempre depende de quem são os indivíduos, da posição que eles ocupam na sociedade e do tipo de sociedade. Um exame mais próximo de secas, terremotos ou enchentes indica que a frequência, potencial de destruição, escopo do impacto etc. estão relacionados com a capacidade social de gerir os desastres (Wenger, 1978).

Essa forma de conceber e analisar os desastres naturais se estabelece na década de cinquenta no contexto da Guerra Fria com o apoio de fundos militares (Quarantelli, 1981). Isso indica que o processo de desenvolvimento inicial da abordagem sociológica dos desastres naturais está relacionado com os interesses militares para com a proteção civil em caso de um ataque nuclear e se concentrou no comportamento de indivíduos e grupos em estresse (Kreps, 1984). As primeiras contribuições que foram realizadas no National Opinion Research Center - NORC da Chicago University (Mark e Fritz, 1954) possuem duas características principais: a) a preocupação com o período pós-impacto; b) a ênfase microssociológica no interacionismo simbólico. Nessas tentativas de sistematização teórica, a atenção se concentra na relação entre normalidade-crise e os desastres naturais são descritos como problemas sociais não-rotineiros que provocam a disrupção do funcionamento da sociedade (Fritz, 1968).

Esses primeiros estudos sobre a relação Estabilidade-Mudança mostraram que era possível produzir conhecimento sociológico sistemático com populações impactadas por desastres naturais. Assim, com a criação do Disasters Research Group, ligado à National Academy of Science - NAS, a implantação do Disasters Research Center na Ohio University em 1963 e, posteriormente, na University of Delaware em 1985, o foco das pesquisas sociológicas sobre desastres naturais foi progressivamente se deslocando para a questão da organização social (Quarantelli, 1994). Mais precisamente, o foco se encontra nas respostas organizadas aos impactos e, dessa forma, os desastres são vistos como um fenômeno concentrado no tempo e no espaço em que a estrutura social é destruída (Pelanda, 1981). Essa forma de análise por meio da consideração da organização social (Webb, 1999) dissemina-se pelo mundo a partir da década de 1970 (Lepointe, 1991).

Nesse sentido, os impactos passam a ser vistos como efeitos da organização social e não afetam as pessoas da mesma forma. Esse pressuposto baseia-se no princípio de continuidade (Quarantelli e Dynes, 1977): a relação que se estabelece entre o período pré-impacto (Tempo 1) e operiodo pós-impacto (Tempo 2). A hipótese subjacente a essa abordagem indica que as condições de vulnerabilidade existentes no Tempo-1 se estendem para o Tempo-2. Isso significa que as condições de vulnerabilidade existentes no período pré-impacto se convertem em destruição no período pós-impacto e que os desastres são um fenômeno inerentemente social, isto é, estão enraizados 
na estrutura social e refletem os processos de mudança social (Perry, 1998). Desse ponto de vista, o desastre natural não é algo conjuntural, com delimitações temporais e espaciais bem definidas, mas exprime as condições sociais de "normalidade" que precedem o impacto (Pelanda, 1981).

A abordagem sociológica dos desastres naturais parte do pressuposto que os eventos são naturais e os impactos são sociais. Portanto, a disputa interdisciplinar estrutura-se numa dupla diferenciação: (a) entre as ciências técnicas e as ciências sociais, por meio da redução da análise das dimensões sociais; (b) no interior das próprias ciências sociais com a geografia, reduzindo o social aos fatores organizacionais. Esse processo está relacionado com duas questões predominantes: (1) a tendência de especialização da comunidade científica norte-americana no período pós-guerra; (2) as mudanças de gerações na concepção dos fatores sociais. A forma como essa diferenciação foi operacionalizada concentrou a atenção do estudo sociológico em disputas teóricas e metodológicas sobre a forma de conceber e analisar o que existe de natural e social nos desastres (Quadro 1). A competição interdisciplinar pode em parte explicar por que a sociologia demorou tanto tempo para estabilizar uma concepção de desastres.

\section{QUADRO 1}

Controvérsia Institucional na Abordagem dos Desastres

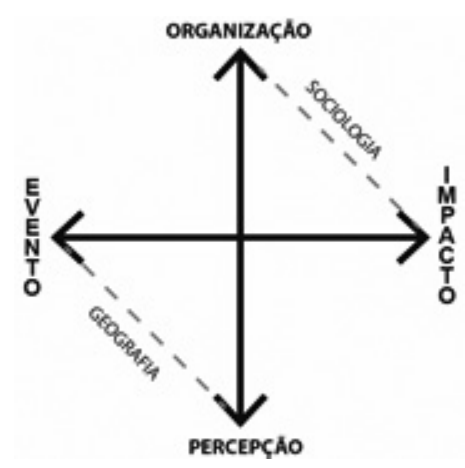

Nessa divisão do trabalho entre a geografia e a sociologia, a natureza é neutra, enquanto a sociedade converte o ambiente em ameaça. As diferenças de abordagem entre a sociologia e a geografia estão relacionadas com dois fatores: a) a ênfase atribuída aos fatores naturais; b) a operacionalização da dimensão social. Enquanto a abordagem geográfica dos hazards foca em como os indivíduos percebem o evento-natural, a abordagem sociológica dos desastres concentra sua atenção nos fatores do impacto-social (conforme Quadro 1). Porém, essa disputa interdisciplinar esconde o fato que as próprias concepções de natural-social são produtos de uma operação 
sociotécnica. O desenvolvimento recente do estudo da ciência e da tecnologia indica que o que entendemos por natural e social também é fixado cientificamente: a natureza neutra e a sociedade interessada. Portanto, a diferenciação entre a geografia e a sociologia com base na ênfase nos fatores naturais e sociais dos desastres impede de perceber o processo de construção sociotécnica dos desastres.

\section{A controvérsia intradisciplinar: a oposição situacional-diferencial}

A controvérsia intradisciplinar diz respeito às disputas que se estabelecem no interior da própria sociologia. Apesar do consenso disciplinar formado sobre a importância de considerar os desastres naturais como um fenômeno social, não se conseguiu ainda construir um acordo teórico sobre como as respostas aos impactos devem ser abordadas, ou seja, quais teorias sociológicas devem ser aplicadas para entender os impactos e qual o seu alcance e abrangência explicativa. (Quarantelli, 1998; Quarantelli e Perry, 2005). Assim, mesmo que se possa afirmar que as condições sociais pré-impacto desempenham um papel decisivo na destruição verificada no período pós-impacto, não existe um acordo sobre que condições sociais são estas e como devem ser analisadas. $\mathrm{O}$ entendimento da controvérsia intradisciplinar pressupõe a consideração de duas dimensões interligadas: (a) a oposição micro-macro - as formas de conceber a vulnerabilidade; (b) a oposição global-local - a localização das formas de conceber as teorias.

A vulnerabilidade constitui a suscetibilidade de um contexto social a impactos causados por desastres naturais (Cutter, 2006). Contudo, não existe consenso no estudo sociológico dos desastres sobre quais são esses fatores sociais. Apesar de existir um acordo sobre a continuidade entre as condições sociais pré e pós-impacto, não existe um consenso sobre a extensão dessas condições sociais, indicando que quanto mais a abordagem sociológica dos desastres se consolida disciplinarmente, menor a unidade paradigmática. Essa diferenciação é produto das incompatibilidades paradigmática e institucional no estudo dos desastres. Portanto, se a abordagem sociológica dos desastres consiste em relacionar os desastres ao contexto social, trata-se de como interpretar essas condições sociais. Assim, podemos considerar duas formas de conceber a vulnerabilidade: (a) a vulnerabilidade situacional; (b) a vulnerabilidade continua.

A vulnerabilidade situacional está relacionada com a abordagem que se estabelece baseando-se nos estudos desenvolvidos no Disaster Research Center (Quarantelli, 1987b). Nesse tipo de abordagem, os desastres são considerados eventos isolados, tendo como protótipo o impacto de um terremoto que é intensivo e concentrado (Fritz, 1968; Quarantelli, 1987b). Essa forma de abordar sociologicamente os de- 
sastres restringe a análise dos impactos ao estudo do comportamento organizado no período de emergência e focaliza o papel desempenhado pela atuação das organizações formais e dos grupos emergentes nos desastres por meio do enfoque realista/ funcionalista (Quarantelli, 2002). Os impactos dos desastres são considerados como contingências situacionais que dependem das condições de organização da comunidade impactada. A organização pré-impacto (mitigação e preparação), vai condicionar o padrão de comportamento pós-impacto (reação e recuperação).

A vulnerabilidade contínua descreve os impactos como resultados de fatores sociais profundos. Os impactos constituem acontecimentos progressivos que são produzidos pelo padrão de desenvolvimento predominante (Clausen et al., 1978; Alexander, 2011). Essa forma de considerar a vulnerabilidade está associada à adoção de quatro perspectivas teóricas que se encontram relacionadas: (a) a crítica da desigualdade do desenvolvimento (Cuny, 1983; Wisner et al., 1994; Maskrey, 1993); (b) a crítica da sustentabilidade ambiental (Kasperson e Kasperson, 2001; Liverman, 1990); (c) a crítica das diferenças sociais (Cutter, 1996; Cutter et al., 2003; Valêncio et al., 2009; Carmo e Valêncio, 2014); (d) a crítica das desigualdade sociais e sua expressão urbana (Wisner, et al., 1994; Ribeiro, 1995; Araujo, 2016). Portanto, na perspectiva diferencial, a vulnerabilidade constitui o resultado de relaçóes sociais desiguais e das representações que os grupos estabelecem dos impactos.

Essa bifurcação exprime duas formas de operacionalização causal do contexto social na explicação dos impactos. Na oposição entre as duas formas de vulnerabilidade situam-se dois tipos de causalidades. Por um lado, os fatores sociais manifestos relativos ao risco de um lugar ser impactado por desastres e, por outro, fatores sociais latentes que estão relacionados com as pré-condiçôes sociais (Cutter, 2005). Dessa forma, as abordagens se diferenciam pela caracterização do ciclo do desastre: (a) na vulnerabilidade situacional, os desastres naturais são descritos por um ciclo restrito que se caracteriza por um início, um meio e um fim muito bem definidos do ponto de vista temporal e espacial (Fritz, 1968; Kreps, 1984); (b) na perspectiva da vulnerabilidade contínua, os desastres naturais são caracterizados como expressão das desigualdades sociais, vistas como consequências de um amplo ciclo político de dominação relativo ao desenvolvimento econômico (Wisner et al., 1994).

Portanto, o estudo dos desastres naturais do ponto de vista sociológico mostra uma mudança de orientação teórica nos últimos anos. Essa mudança de concepção indica que o tipo de desastre estudado no passado não é o mesmo estudado no presente. Assim, os desastres naturais deixaram de ser vistos como problemas sociais não rotineiros (Hart e Kreps, 1986), e passaram a ser entendidos como fenômenos comuns que refletem as características do contexto social em que ocorrem (Tierney, 2006, 2007). Esse fato tem sido descrito de diversas formas, mas os traços predo- 
minantes revelam que os processos de integração e participação social em desastres passaram a ser descritos com base nos padrões de interação que se estabelecem entre indivíduos e grupos. $\mathrm{Na}$ passagem da plataforma teórica da vulnerabilidade situacional para a plataforma da vulnerabilidade continua, verifica-se o progressivo deslocamento da atenção da "vulnerabilidade" para os "vulneráveis".

QUADRO 2

Formação do Campo Disciplinar

\begin{tabular}{|c|c|c|c|c|}
\hline COMPONENTES FASES & \begin{tabular}{|c|}
$\begin{array}{c}\text { Fase de implantaç3o } \\
\text { (1952-1962) }\end{array}$ \\
\end{tabular} & \begin{tabular}{|c} 
Fase de consolidac Jio \\
(1963-1985) \\
\end{tabular} & 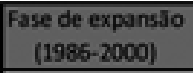 & $\begin{array}{c}\text { Fase de diversificação } \\
\text { (2000-2015) } \\
\end{array}$ \\
\hline \multirow{3}{*}{ Expoentes } & C. Fritz & E. L. Quarantelli & W. Dombrowsky & K. J. Tierney \\
\hline & A. Wallace & R. Dynes & R. Stallings & N. Venalincio \\
\hline & 5. Star & G. Kreps & A. Lavell & 5. Revet \\
\hline Instituiç̧ొం & NORC - DRG & DCR & LARED & ISDR \\
\hline Teorias & $\begin{array}{l}\text { Interacionismo } \\
\text { simbólico }\end{array}$ & Funcionalismo & Neo-Marxismo & Construtivismo \\
\hline Objeto & $\begin{array}{c}\text { Situaçăo de crise - } \\
\text { vitimas }\end{array}$ & $\begin{array}{l}\text { Organizapðes } \\
\text { emergentes }\end{array}$ & Vulnerabilidade & Vulneraiveis \\
\hline
\end{tabular}

Nesse sentido, a intensidade da Controvérsia Intradisciplinar encobre uma luta feroz pela influência cognitiva (Bourdieu, 1975; Latour, 1994). Trata-se da capacidade de transformar subvenções em equipamentos, equipamentos em dados, dados em argumentos, argumentos em artigos etc. Portanto, conforme Quadro 2, entre a primeira e quarta geração de pesquisadores em sociologia dos desastres opera-se uma deslocalização e uma intensificação da competição pela credibilidade. Dito de outra forma, à medida que a sociologia dos desastres se internacionaliza, os dados não são mais os mesmos, os argumentos se diferenciam, os artigos aparecem em outras revistas e, dessa forma, os dados se tornam mais difíceis de serem comparados, os argumentos de serem entendidos, os artigos de serem revisados. Para entender essa competição temos que visitar os pontos da rede em que as teorias são concebidas: (a) mediação global-grupos geradores de teorias; (b) mediação local - grupos propagadores de teorias.

A mediação global se forma e se propaga com os grupos de pesquisa pioneiros formados nos Estados Unidos nos anos de 1960 no Disasters Research Center. A partir dos anos de 1970 se dissemina, de uma maneira mais institucionalizada, para a Europa, na Alemanha (Katastrophenforschungsstelle - Uni Kiel), na Itália (Istituto di Sociologia Internazionale di Gorizia) e, principalmente, na Inglaterra (Disaster Research Unit - University of Bradford) etc. Opõe-se, dessa maneira, a abordagem americana à europeia. A influência desse grupo de pesquisa se exprime na instituição e controle do Research Committee on Sociology of Disasters RC39 da ISA, e do principal periódico o International Journal of Mass Emergencies and 
Disasters. Porém, apesar das diferenças, os desastres nos países do Terceiro Mundo são pesquisados por esses sociólogos sem referências aos autores da região (Kreps, 1995; Wisner, 1994) ou, mesmo quando as concepções locais são consideradas, elas são feitas por porta-vozes centrais (Mercer et al., 2010).

A mediação local se estabelece valendo-se do processo de deslocalização da sociologia dos desastres na década de 1970. Com o processo de disseminação da sociologia dos desastres, principalmente com base na América Latina, desencadeia-se um questionamento da adequação da abordagem standard nos termos da crítica da dependência acadêmica (Gareau, 1988; Alatas, 2003). Assim, com base em experiências de pesquisa como as desenvolvidas, principalmente, por La Red de Estudios Sociales en Prevencion de Desastres en America Latina e a revista Desastres y Sociedad, estabelece-se um padrão de tradução local dos desastres (Lavell, 2004). Dessa forma, a mediação local indica, por um lado, o reconhecimento da aplicabilidade limitada da sociologia dos desastres mainstream; e, por outro, a necessidade de construir um conhecimento territorialmente localizado e socialmente heterogêneo dos desastres como problemas sociais não resolvidos (Valêncio et al., 2009; Carmo e Valêncio, 2014).

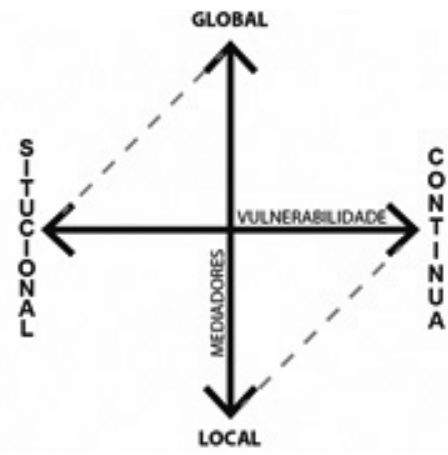

A controvérsia intradisciplinar exprime a dificuldade dos mediadores globais de disciplinar a abordagem sociológica dos desastres. A disputa opõe o eixo hegemônico, que liga a concepção dos impactos dos desastres, relativos à vulnerabilidade situacional, às formas de mediação global, ao eixo que relaciona a vulnerabilidade contínua às formas de mediação local, conforme explicita o Quadro 3. Enquanto a controvérsia se restringia a um pequeno grupo de sociólogos norte-americanos, as diferenças se restringiam a teorias e métodos, mas à medida que começam a participar sociólogos europeus, latino-americanos, africanos e asiáticos, começam a surgir pontos de resistência aos compromissos fixados. O questionamento e a redefinição dos contornos disciplinares indicam a incapacidade dos mediadores 
globais de convencer e interessar os mediadores locais sobre a adequação do repertório sociológico: quanto mais a rede disciplinar se estende, menos homogênea se torna a abordagem.

\section{A controvérsia extradisciplina: a oposição recuperação-preparação}

A controvérsia extradisciplinar constitui o resultado das conexões exteriores que a sociologia dos desastres estabelece com o contexto social. A pesquisa sociológica sobre desastres nunca foi um fim em si mesma (Stallings, 1998). Afinal, desde o início sempre teve uma forte ligação com questões práticas relacionadas com a gestão dos desastres. Mais precisamente, a sociologia dos desastres sempre manteve um vínculo direto com questões governamentais, inicialmente com a Defesa Civil (Knowles, 2011) e posteriormente com a formulação e implementação de políticas públicas (Waugh Jr, 2000). A interação com uma grande variedade de atores afeta o conteúdo dos enunciados e a orientação da abordagem disciplinar. Essa contextualidade indica o caráter heterogêneo da composição da sociologia dos desastres. Emerge, com isso, a questão das negociações e engajamentos relacionados com a aplicabilidade da expertise sociológica dos desastres como um artefato sociotécnico.

As disputas relacionadas com a transformação da abordagem sociológica dos desastres num artefato sociotécnico estão associadas a dois fenômenos predominantes. Por um lado, progressivamente os impactos são confrontados por organizações especializadas que transformam os desastres no centro de suas operações; por outro, começa a se estabelecer um conjunto de profissionais, experts e técnicos especializados na gestão dos desastres. Por isso, o desenvolvimento da abordagem sociológica dos desastres envolve também a consideração dos conjuntos de negociações relativas à adequação e utilização do conhecimento sociológico na gestão dos desastres; e, consequentemente, envolve as disputas relativas à aplicação da prática da sociologia dos desastres (Trainor e Subbio, 2014). A compreensão dos conflitos relacionados com a aplicabilidade da sociologia dos desastres pressupõe a consideração de duas dimensões principais: (a) os tipos de intervenção; (b) os padröes de expertise.

Os tipos de intervenção diferenciam-se em função das fases dos desastres (antes, durante e depois) (Carr, 1932; Wallace, 1956; Clausen, 1992; Stallings, 1998). Frequentemente as medidas são divididas em quatro fases: (a) pré-impacto - mitigação e preparação; (b) trans e pós-impacto - resposta e recuperação. A mitigação constitui ações pré-desastre que visam eliminar ou diminuir os riscos de ocorrência de um evento; a preparação refere-se às açôes de planejamento que visam antecipar a ocorrência do evento com medidas de previsão ou treinamento; a resposta diz respeito ao processo de reação e operações de salvamento durante a ocorrência do evento; e, 
por último, a recuperação compreende atividades destinadas ao reestabelecimento das condiçôes básicas das redes de serviços e abrigos temporários (Waugh Jr., 2000). Assim, nas últimas décadas verifica-se uma mudança de perspectiva das ações pós-impacto para as ações pré-impacto.

Apesar do deslocamento do foco de intervenção das medidas pós-impacto para as medidas pré-impacto, o debate sobre a implementação permanece controverso (Revet, 2009). Esse modelo foi concebido nos Estados Unidos pela Federal Emergence Management Agency - FEMA durante a década de 1970 e, posteriormente, disseminado para outros países. Com a criação da United Nations Disasters Relief Organization - UNDRO, em 1971, a questão da gestão dos desastres e das medidas de preparação se internacionaliza. A questão no estabelecimento de ações de preparação envolve a substituição da ênfase no socorro pela concepção de aprender a conviver com o risco (ISDR, 2004). Mais precisamente, opõe, de um lado, estratégias centralizadas de viés tecnocrático e, de outro, estratégias descentralizadas baseadas na comunidade. Ainda que não possam ser consideradas homogêneas, duas perspectivas se diferenciam: (a) gestão tecnocrática; (b) gestão comunitária.

A gestão tecnocrática ou a prevenção top-down está relacionada com o crescente papel desempenhado pelo Estado e pelas agências internacionais na gestão dos desastres. Essa forma de conceber o processo de gestão dos desastres converte as comunidades e as pessoas em vítimas passivas dos impactos dos desastres. Nessa concepção dos desastres enfatiza-se a necessidade, urgência e trauma dos impactos, e a necessidade de conceber medidas de confrontação. Aqui, claro, trata-se de reafirmar o papel central da ciência e da tecnologia para prevenção dos desastres naturais e a importância da informação para proteção das populações locais. Nesse padrão de gestão, encontra-se subsumida uma concepção de segurança que centra as ações no individuo e em suas necessidades vitais. Portanto, diante de um cenário de ameaça constante existiriam alguns kits políticos e tecnológicos que permitiriam reduzir o risco dos desastres por meio da implementação de ações de prevenção (Revet, 2011).

Essa dimensão da controvérsia extradisciplinar está relacionada com a questão política da sociologia dos desastres. Enquanto as medidas tecnocráticas visam prioritariamente o salvamento de vidas no momento de impacto, as medidas comunitárias visam o fortalecimento da capacidade de resiliência. Em outras palavras, temos a oposição entre operações de socorro de curto prazo, de cima para baixo, e medidas que privilegiam as ações de longo prazo em períodos de não-impacto. Da oposição entre esses dois tipos de medidas, emerge a questão da centralização e da descentralização e também a questão da expansão ou contração da participação da comunidade. Aqui, trata-se de verificar como o poder encontra-se redistribuído e como as estratégias de gestão se convertem em mecanismos de dominação. Portanto, o que está em jogo na 
gestão dos desastres é a manutenção das relações sociedade-Estado e, assim, o papel da sociologia dos desastres diante das formas de dominação.

Além disso, a implementação dessas medidas envolve um arranjo institucional muito complexo e mobiliza interesses de atores muito diversos. A gestão dos desastres aciona agências das esferas federal, estadual e municipal; inclui também serviços de caridade e organizações não governamentais; além disso, é preciso considerar também as organizações privadas que prestam serviços para o governo; bem como a sociedade civil organizada, os movimentos sociais e as organizações emergentes; e em algumas situações também agências internacionais como a ISDR da ONU ou a Cruz Vermelha. A responsabilidade e a capacidade são diferentes, o que sempre acaba atraindo diferentes tipos de atores como, por exemplo, vítimas, voluntários, técnicos, burocratas, jornalistas, políticos, pesquisadores etc. Por isso, os interesses envolvidos na gestão dos desastres são divergentes e, muitas vezes, até conflitantes. Verifica-se, portanto, diversos padrões de expertise na gestão dos desastres.

A questão da expertise em geral (Collins e Evans, 2007; Collins, 2014) e da expertise em gestão dos desastres tem sido objeto de muitos debates (Trainor e Subbio, 2014). Esse conhecimento constitui a mistura entre relatórios técnicos e análise social. Por um lado, o conhecimento sociológico produzido é incorporado por especialistas e técnicos e afeta o processo de gestão dos desastres (Dynes, 1994); por outro, subsidia a organização da sociedade e fomenta a resistência à centralização e tecnocracia governamental (Enarson et al., 2010). A abordagem sociológica dos desastres constitui também o resultado de uma negociação política com interesses de ordem teórica e prática. Por isso, a questão da expertise constitui um aspecto central na consideração da abordagem sociológica dos desastres. Nesse sentido, é possível diferenciar dois tipos de competências na gestão dos desastres: (a) expertise técnica; (b) expertise relacional.

A expertise técnica constitui o resultado do processo de treinamento sistemático na tecnociência, legitima-se em argumentos técnicos fundamentados em vários indicadores como, por exemplo, o Disaster Vulnerability Index (DVI), o Disaster Risk Indicators (DRI), o Global Risk and Vulnerability Index Trends (GRAVITY), o Natural Hazards Apparent Vulnerability Indicator (NHAVI) etc. (Revet, 2011) e é exercida por profissionais, principalmente em organizações governamentais especializadas que convertem os desastres em centro de atuação (Defesa Civil, Bombeiros, agências governamentais etc.). Trata-se de especialistas responsáveis pela implementação das políticas públicas e programas de proteção a desastres, entre os quais se destacam diretores, planejadores, consultores, técnicos etc. A experiência acumulada indica que a expertise técnica caminha para a profissionalização da gestão dos desastres (Trainor e Subbio, 2014). 
A expertise relacional constitui o conhecimento adquirido por comunidades, grupos ou indivíduos por meio do convívio prático com o problema dos desastres. Trata-se de um conhecimento tácito incorporado com base na experiência de convivência com a recorrência do problema dos desastres. Esse aprendizado diz respeito à capacidade de a comunidade local gerir o processo de concepção e implementação de medidas de redução de risco de desastres. Nesse sentido, verifica-se o reconhecimento dos mecanismos existentes de enfrentamento e das capacidades da comunidade/pessoas, bem como do know-how e dos recursos locais para estabelecer estratégias e planos de redução de risco de desastre, priorizando a participação dos grupos mais vulneráveis. Esse reconhecimento exprime a crescente conscientização do valor do conhecimento prático como, por exemplo, a associação do conhecimento tradicional e indígena ao conhecimento científico na redução do risco de desastres (Mercer et al., 2010).

A interação entre expertise técnica e expertise relacional é marcada por diversos pontos de tensão no estabelecimento de normas, standards de boa gestão, indicadores de vulnerabilidade, tecnologias de observação dos fenômenos, glossários etc. As formas de apropriação e aplicação do conhecimento sociológico dos desastres encerram, assim, diversas ordens de disputas, conforme Quadro 3. Por um lado, emerge a questão do papel da sociologia dos desastres na disputa pelo monopólio e autoridade do conhecimento sobre os desastres; por outro, destaca-se a questão do controle e dominação que se exprime na crescente centralização dos processos de gestão. Nesse contexto, verifica-se que os indivíduos impactados têm cada vez menos controle sobre si mesmos, diante de um crescente processo de tecnificar, profissionalizar e internacionalizar a gestão dos desastres (Revet, 2011; Cwiak e Goss, 2014).

QUADRO 4

Controvérsia Politica na Gestão dos Desastres

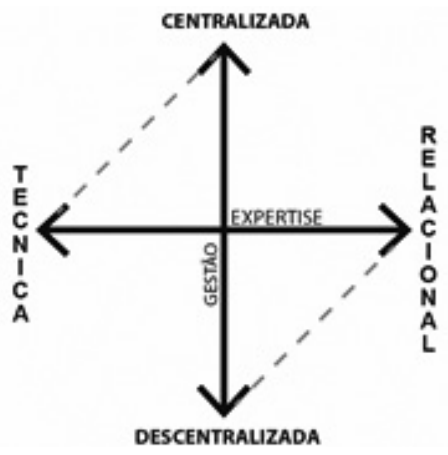

A controvérsia extradisciplinar sobre a adequação das medidas sociotécnicas de gestão tem muitas consequências para abordagem sociológica dos desastres. No eixo medidas tecnocráticas - expertise técnica pressupõe-se a gestão dos desastres como um 
processo neutro e consensual produzido por um acordo tácito. Assim, em contextos sociais impactados por desastres prevalecem normas altruísticas e as diferenças sociais são temporariamente suspensas devido ao "efeito nivelamento" (Dynes e Quarantelli, 1975; Quarantelli et al., 1998), porque são considerados contextos sociais politicamente neutros (Dynes, 1994). No eixo medida comunitárias - expertise relacional a gestão dos desastres é pensada como um processo conflitivo. Esse processo indica o acesso diferencial aos recursos que se exprime socialmente em termos de classes sociais, etnia, gênero, idade etc. (Phillips e Fordham, 2010) ou, em outros termos, "ganhadores" e "perdedores" (Scanlon, 1988).

Uma nova agenda para a pesquisa dos desastres

A comunidade de pesquisadores em sociologia dos desastres efetuou importantes descobertas estudando as respostas aos impactos dos desastres naturais. Os esforços da primeira geração de pesquisadores concentraram-se na demarcação disciplinar: justificar a importância da abordagem sociológica dos impactos dos desastres naturais. Já a segunda geração esteve mais preocupada com a consolidação disciplinar: institucionalizar uma rede de pesquisa sociológica em desastres. Na terceira geração, a atenção foi para a integração da sociologia dos desastres dentro do campo sociológico: o estabelecimento de uma teoria compativel com o desenvolvimento do mainstream disciplinar. A quarta geração, por fim, enfatizou a diversificação: a determinação de para quem serve a sociologia dos desastres. Verifica-se, portanto, que à medida que a comunidade de pesquisadores em sociologia dos desastres foi se institucionalizando a cultura disciplinar foi se diferenciando.

Nesse sentido, as controvérsias que marcam o desenvolvimento da sociologia dos desastres refletem as transformações da organização, entendimento e gestão dos desastres nas últimas décadas. Por um lado, essas transformações possibilitaram uma compreensão mais precisa do fenômeno; por outro, modificaram também a forma de convívio com o problema. O exame das controvérsias indica que a abordagem sociológica dos desastres naturais possui três características principais: (1) disciplinarmente flexivel; (2) teoricamente eclética; (3) politicamente conflitiva. Portanto, é possível extrair três implicações operacionais para a sociologia: (1) a sociologia dos desastres não é feita somente por sociólogos; (2) não é possivel demarcar um conjunto de abordagens especificas; (3) a sociologia dos desastres possui um viés deliberadamente aplicado. Esse processo indica que a sociologia dos desastres não é apenas multidisciplinar, mas também multidimensional e multissetorial.

Reconhecer que os desastres constituem um fenômeno multidimensional, multidirecional, multissignificativo implica assumir o caráter eminentemente controverso 
da abordagem sociológica dos desastres. Nesse contexto podemos retomar os três paradoxos que marcam o desenvolvimento da sociologia dos desastres: (1) oparadoxo da informação: o aumento da naturalização dos desastres esconde o caráter tecnocientífico dos impactos; (2) o paradoxo da intensificação: o aumento de pesquisadores intensifica as disputas teóricas; (3) o paradoxo da gestão: o aumento da capacidade de confrontação estimula a ocupação de áreas de risco. Um novo programa para a sociologia dos desastres deve se concentrar na crítica da ideologia dos desastres: as disputas cognitiva e política que se estabelecem na oposição entre a naturalização e a culturalização dos desastres.

Portanto, para responder à questão "o que é um desastre?" precisamos olhar para dentro e para fora da sociologia. Mais precisamente, precisamos considerar quais são as semelhanças e diferenças que a sociologia estabelece com as outras disciplinas, mas também como esse conhecimento é utilizado socialmente. Isso significa que a abordagem sociológica dos desastres deve ser precedida por uma abordagem sociológica do conhecimento dos desastres, de como o conhecimento dos desastres, inclusive o conhecimento produzido pela própria sociologia dos desastres, relaciona-se com o contexto social. Para isso é preciso dotar a sociologia dos desastres de uma disposição reflexiva: considerar como a ciência em geral e a sociologia em particular contribuem para fixar os processos de "naturalização" ou "culturalização" dos desastres. Nesse sentido, um desastre constitui a dissolução da rede sociotécnica que associa simbólica e materialmente o mundo social ao mundo natural.

Uma das implicações pouco exploradas dessas transformações diz respeito à própria naturalidade dos desastres naturais. $\mathrm{O}$ exame atento do processo de formação e desenvolvimento da abordagem sociológica dos desastres indica que a preocupação com a dimensão natural (evento) foi dando lugar aos fatores sociais (impacto). Essa tendência é, ao mesmo tempo, cognitiva e política, e se exprime de duas formas: a) a centralidade da teoria da vulnerabilidade: (a) centralidade assumida pelo conceito de vulnerabilidade nos últimos anos; (b) protagonismo das políticas de preparação: a ênfase nas ações pré-impacto nos processos de gestão dos desastres. Nesse sentido, o questionamento da naturalidade dos desastres inscreve-se no processo de problematização construtivista da manipulação sociotécnica das relações entre sociedade e natureza. Por isso, talvez, fosse mais adequado substituir o adjetivo natural na abordagem sociológica dos desastres.

Assim, os desastres podem ser considerados uma ferramenta metodológica de estudo da sociedade. Afinal, desenvolver uma teoria sociológica dos desastres naturais compreende desenvolver uma teoria do contexto social impactado. Por ser uma ruptura, os desastres permitem entender como o contexto social é ordenado por meio de rotinas e associações. Assim, a definiçãa de desastre depende da forma 
como concebemos cognitivamente e manipulamos tecnicamente o mundo. $\mathrm{Ou}$ seja, a disrupção das rotinas sociais provocadas por um desastre natural depende de como concebemos o contexto social. Isso significa que os desastres precisam se analisados de forma simétrica; não se trata de observar o contexto social para entender os desastres, mas precisamos inverter a relação: observar os desastres para entender $o$ contexto social. Portanto, o impacto dos desastres permite examinar a composição do contexto social.

No que se refere à abordagem dos desastres em língua portuguesa, a produção parece acompanhar as tendências recentes. Por muito tempo a questão dos desastres permaneceu fora da agenda de pesquisa mainstream tanto em Portugal quanto no Brasil. Esse processo está relacionado com dois fatores que se encontram interligados: (a) uma falha de registro decorrente de uma autoimagem nacional segunda o qual desastres não eram recorrentes; (b) as características específicas da formação do campo sociológico no Brasil e em Portugal. Nesse sentido, apesar da centralidade da temática na formação histórica dos países como o terremoto de Lisboa ou a secas no Nordeste Brasileiro, a questão dos desastres permaneceu fora da agenda. Somente a partir da década de 1990, num contexto político e acadêmico marcado pelo processo de redemocratização e internacionalização da comunidade científica, a questão dos desastres passa a integrar a agenda de pesquisa, financiamento e publicação.

\section{Referências Bibliográficas}

Alatas, S. F. (2003), "Academic Dependency and the Global Division of Labour in the Social Sciences". Current Sociology, 6 (51): 599-613.

Alexander, D. (2000), Confronting catastrophe. Harpenden, Oxford University Press. . (2005), "An interpretation of disaster in terms of changes in culture, society and international relations". In: PERRY, R. W. \& QUARANTELLI, E. L. (orgs). What is a disaster? New answers to old questions. (s.l.), International Research Committee on Disasters, pp. 25-38. . (2011), "Modelos de vulnerabilidade a desastres". Revista Crítica de Ciências Sociais, 93: 9-20.

Araujo, P. (2016), "Esboço de uma sociologia com desastres: o caso da tragédia de Entre-os-Rios". Análise Social, 218, LI (1º): 72-95.

Bankoff, G. et al. (2011), Mapping vulnerability: disasters, development \& people. Sterling, Earthscan.

BECK, U. (1992), Risk society: towards a new modernity. Trowbridge, Sage.

Bloor, D. (1991), Knowledge and social imagery. Chicago, University of Chicago Press.

Bourdieu, P. (1975), La spécicificite du champ scientifique et les conditions sociales du progress de la raison. Sociologie et Societés, 1 (7): 91-188. 
Bryant, E. A. (1991), Natural hazards. Nova York, Cambridge University Press.

Burton, I. et al. (1993), The environment as hazards. Nova York, The Guilford Press.

Callon, M. (1986), "Éléments por une sociologie de la traduction: la domestication des coquilles Saint-Jacques et des paris-pêcheurs dans la baie de Saint-Brieuc”. Anné Sociologieque, 36: 169-208.

Carmo, R. \& ValÊncio, N. (2014), Segurança humana no contexto de desastres. São Carlos, RiMa.

Carr, L. J. (1932), “Disaster and the Sequence-Pattern Concept of Social Change”. American Journal of Sociology, 2 (38): 207-218.

Cetina, K. K. (1999), Epistemic cultures: how the sciences make knowledge. Cambridge, Harvard University Press.

Clausen, L. (1992), "Social differentiation and the long-term origin of disasters". Natural Hazards, 6: 181-190.

Clausen, L. et al. (1978), "New aspects of the sociology of disasters: theoretical note". Mass Emergencies, 3: 61-65.

Collins, H. (1992), Changing order: replication and induction in scientific practice. Chicago, The University Chicago Press.

\& Evans, R. (2007), Rethinking expertise. Chicago, University of Chicago Press.

Collins, R. (1994), Four sociological traditions. Oxford, Oxford University Press. . (2014), Are we all scientific experts now? Cambridge, Polity Press.

Crossley, N. (2011), Towards relational sociology. Nova York, Routledge.

Crowe, A. (2012), Disasters 2.0: the application on social media systems for modern emergency management. Boca Raton, Taylor \& Francis Group.

Cuny, F. C. (1983), Disasters and development. Oxford, Oxford University Press.

Cutter, S. L. (1996), "Vulnerability to environmental hazards”. Progress in Human Geography, 4 (20): 529-539.

. (2005), "The geography of social vulnerability: race, class, and catastrophe. Understanding Katrina: perspectives from the social sciences". Social Sciences Research Council. Disponível em http://understandingkatrina.ssrc.org/Cutter/.

. (2006), "Vulnerability to environmental hazards". In: . Hazards, vulnerability and environmental justice. Sterling, Earthscan, pp. 71-82.

et al. (2003), "Social vulnerability to environmental hazards". Social Science Quarterly, $2(84): 242-261$.

Cvet kovich, G. \& Earle, T. C. (1985), “Classifying hazardous events”. Journal of Environmental Psychology, 5: 5-35.

Cwiak, C. L. \& Goss, K. C. (2014), “Prossionalization”. In: Trainor, J. E. \& Subbio, T. Critical issues in disaster Science and management: a dialogue between researchers and practitioners. Washington, Fema, pp. 393-426. 
DAgOn, M. \& PAHRe, R. (1991), L'innovation dans les sciences sociales: la marginalitécréatrice. Paris, Presses Universitaires de France.

Dombrowsky, W. R. (1998), “Again and again: is disaster what we call a 'disaster'?” In: QUARANTElli, E. L. What is a disasters? Perspectives on the question. Nova York, Routledge, pp. 19-30.

Donati, P. (2011), Relational sociology: a new paradigm for social sciences. Nova York, Routledge.

Douglas, M. \& Wildavsky, A. (1982), Risk and culture: an essay on the selection of technological and environmental dangers. Los Angeles, University of California Press.

DRABEK, T. E. (1986), Human system response to disaster: an inventory of sociological findings. Harrisonburg, Spinger.

Dynes, R. R. (1994), “Conceptualización del desastre en formas productivas para la investigación em ciências sociales”. In: LAWELL, Allan. Al norte del rio Grande - ciencias sociales, desastres: uma perspectiva norteamericana. Bogotá, La Red, pp. 127-154.

\& QuARANTELLI, E. L. (1975), Community conflict: its absence and presence in natural disasters. Newport, Disaster Center Research - Preliminary Paper 18.

Eckeberg P. L. \& Hill, L. (1979), “The paradigm concept and sociology: a critical review”. American Sociological Review, 6 (44): 925-937.

Enarson, E. et al. (2010), "New ideas for practitioners". In: Phillips, B. et al. Social vulnerability to disasters. Boca Raton, CRC Press, pp. 345-365.

Fritz, C. E. (1961), “Disasters”. In: Merton, R. \& Nisbet, R. (eds.). Social problems. Nova York, Harcourt Brace.

. (1968), “Desastres”. In: Sills, D. L. Enciclopedia Internacional de las Ciencias Sociales. Madri, Aguilar, vol. 3, pp. 618-632.

Gareau, F. H. (1988), “Another type of third world dependency: the social science”. International Sociology, 2 (3): 171-178.

Girdens, A. (2009), A constituição da sociedade. São Paulo, Nova Fronteira.

GIlbert, C. (1995), "Studying disaster: changes in the main conceptual tools”. International Journal of Mass Emergencies and Disasters, 3 (13): 231-240.

Guggenheim, M. (2014), “Introduction: disasters as politics - politics as disasters". Sociological Review, 62 (S1): 1-16.

Hart, O. D. \& Kreps, D. (1986), “Price Destabilizing Speculation”. Journal of Political Economy, 94 (5): 927-952.

Heijmans, A. (2009), The social life of community-based disaster risk reduction: origins, politics and framing. Bolder, Disaster Studies Working Paper 20 - Hazard Research Centre.

Hewitt, K. \& Burton, I. (1971), The hazardousness of place: a regional ecology of damaging events. Toronto, University of Toronto Press.

Hewitt, K. (1983), “The idea of calamity in a technocratic age”. In: (org.). Interpretations of calamity: from the view point of human ecology. Londres, Allen \& Unwin, pp. 3-32. 
ISDR. (2004), Living with risk: a global review of disaster reduction initiatives. Genebra, United Nations - ISDR.

KASPERson, J. X. \& KASPERSON, R. E. (2001), International workshop on vulnerability and global environmental change. Stockholm, Stockholm Environment Institute.

Kates, R. (2011), Gilbert White 1911-2006: a biographical memoir. Washington, D.C., National Academy of Sciences.

Knowles, S. G. (2011), The disasters experts: mastering risk in modern America. Philadelphia, University of Pennsylvania Press.

KozÁx, J. \& CERMÁx, V. (2010), The illustrated history of natural disasters. Nova York, Springer. Kreps, G. A. (1984), “Sociological inquiry and disasters research”. Annual Review Sociological, 10: 309-330. (ed.). (1989), Social structure and disaster. Newark, University of Delaware Press. . (1995), "Disaster as Systemic Event and Social Catalyst". International Journal of Mass Emergencies and Disasters, 3 (13): 255-284.

Kunn, T. (1989), A estrutura das revoluções cientifica. São Paulo, Perspectiva.

Latour, B. (1994), Jamais fomos modernos: ensaio de Antropologia simétrica. Rio de Janeiro, Editora 34 .

. (1999), Politiques de la nature. Comment faire entrer les sciences en démocratie. Paris, La Découverte.

. (2000), Ciência em ação: como seguir cientistas e engenheiros sociedade afora. São Paulo, Editora da Unesp.

LAVELL, A. (1994), Al norte del río Grande - ciencias sociales, desastres: una perspectiva norteamericana. Bogotá, La Red.

- (2004), La red de estudios sociales en prevención de desastres en américa latina, la red: antecedentes, formación y contribución al desarrollo de los conceptos, estudios y la práctica en el tema de los riesgos y desastres en América Latina: 1980-2004. Lima, La Red.

Lemaine, G. et al. (1976), Perspectives on the emergence of scientific disciplines. Paris, Maison des Sciences de L'Homme.

Lepointe, E. (1991), "Le sociologie et les dèsastres". Cahiers Internationaux de Sociologie, v. XC: $145-174$.

Liverman, D. (1990), "Vulnerability to global environmental change”. In: Kasperson, R. E. et al. (ed.) Understanding global environmental change: the contributions of risk analysis and management. Worcester, The Earth Transformed Program, pp. 27-44.

Luhmann, N. (1992), Sociologia del riego. Jalisco, Universidad Iberoamericana/Universidad de Guadalajara.

Maskrey, A. (1993), Los desastres no son naturales. San José, La Red.

MARK, E. S. \& Fritz, C. E. (1954), “The NORC Studies in human behavior in disaster”. Journal of Social Issues, 10: 26-41. 
Mattedi, M. A. \& Butzke, I. C. (2001), "A relação entre o social e o natural nas abordagens de hazards e de desastres". Ambiente \& Sociedade, 9 (Iv): 1-23.

Mercer, J. et al. (2010), "Framework for integrating indigenous and scientific knowledge for disaster risk reduction”. Disasters, 1 (34): 214-239.

Merton, R. K. (1996), On social structure and science. Chicago, The University of Chicago Press.

Montz, B. E. \& Toвin, G. A. (2011), "Natural hazards: an evolving tradition in applied geography". Applied Geography, 31: 1-4.

Pantti, M. et al. (2012), Disasters and the media. Nova York, Peter Lang.

Peduzzi, P. et al. (2009), "Assessing global exposure and vulnerability towards natural hazards: the Disaster Risk Index". Natural Hazards and Earth System Sciences, 9: 1149-1159.

Pelanda, C. (1981), Disaster and sociosystemic vulnerability. Newark, Preliminary Paper 68 - Disaster Research Center/University of Delaware.

Perry, R. (1989), “Taxonomy classification, and theories of disaster phenomena,” In: KrePs, G. (ed.), Social structure and disaster. Newark, University of Delaware Press.

. (1998) "Definitions and the development of a theoretical superstructure for disaster research". In: QuARANTelli, E. L. (ed.), What is a disaster? Perspectives on the question. Londres, Routledge, pp. 197-215.

. (2006), "What's a disaster?” In: Rodríguez, H. et al. Handbook of disaster research. Nova York, Springer, pp.1-15.

Phillips, B. \& Fordham, M. (2010), “Introduction”. In: Phillips, B. et al. Social vulnerability to disasters. Boca Raton, CRC Press, pp. 1-23.

Popper, K. R. (2006), A lógica da pesquisa cientifica. São Paulo, Cultrix.

Porfiriev, B. N. (1995), "Disaster and disasters areas: methodological issues of definition and delineation”. International Journal of Mass Emergencies and Disasters, 3(13): 285-304.

PrINCE, S. H. (1920), Catastrophe and social change: based upon a sociological study of the Halifax disaster. Nova York, doctoral dissertation, Columbia University, Department of Political Science.

Quarantelli, E. L. (1978), Disasters: theory and research. Beverly Hills, Sage. . (1981), "The description and evaluation of the DRC work in the disaster area". Disaster Research Center - Preliminary Paper 65.

_. (1987a), "What should we study? Questions and suggestions for researchers about concept of disasters". International Journal of Mass Emergencies and Disasters, 1 (5): 7-32. . (1987b), "Disaster studies: an analysis of the social historical factors affecting the development of research in the area". International Journal of Mass Emergencies and Disasters, 3 (5): 285-310.

. (1989), "Conceptualizing disasters from sociological perspective," Internacional Journal of Mass Emergencies and Disasters, 7: 285-310.

. (1994), "Disasters studies: the consequences of the historical use of a sociological 
approach in the development of research". International Journal of Mass Emergencies and Disasters, 1 (12): 25-49.

. (1998), What is a disasters? Perspectives on the question. Nova York, Routledge.

. (2002), "The Disaster Research Center (DRC) filed studies of organized behavior in the crisis time period of disasters". In: STALLINGS, R. A. Methods of disasters research. Palo Alto, International Research Committee on Disasters.

\& Dynes, R. (1970), "Property norms and looting: their pattern in community crises". Phylon, 31: 168-182.

$\&$ . (1977), "Response to Social Crisis and Disaster". Annual Review of Sociology, 3: 23-49

\& PERry, R. W. (2005), What is a disasters? New answers to old questions. (s.l.), International Research Committee on Disasters.

Quarantelli, E. L. et al. (1998), "A heuristic approach to future disasters and crises: New, Old, and In-Between Types". In: Quarantelli, E. L. What is a disasters? Perspectives on the question. Nova York, Routledge, pp. 16-41.

Revet, S. (2009), "Vivre dans un monde plus sûr: catastrophes 'naturelles' et sécurité 'globale”". Cultures \& Conflits, 75: 33-51.

. (2011), "El mundo internacional de las catástrofes naturales". Política y Sociedad, 3 (48): $537-554$.

Ribeiro, J. M. (1995), “Sociologia dos desastres”. Sociologia: Problemas e Práticas, 18: 23-43.

Rodríguez, H. et al. (2006), Handbook of disaster research. Nova York, Springer.

SCANLON, L. (1988), "Winners and losers: some thoughts about the political economy of disasters". International Journal of Mass Emergencies and Disasters, 6: 47-63.

SCANDLYN, J. et al. (2010), “Theoretical framing of worldviews, values, and structural dimensions of disasters". In: PhILlips, Brenda D. et al. Social vulnerability to disasters. Boca Raton, CRC Press.

Schenk, G. J. (2007), "Historical disasters research. State of research, concepts, methods and the studies". Historical Social Research, 3 (32): 9-31.

Seidman, S. (2004), Contested knowledge: social theory today. Oxford, Blackwell.

SHI, P. \& KASTERSON, R. (2015), World atlas of natural disasters risk. Worcester, BNUP/Springer.

Stallings, R. A. (1998), "Disaster and the theory of social order". In: QUaRANTElli, E. L. What is a disasters? Perspectives on the question. Nova York, Routledge, pp. 127-145.

Stichweh, R. (1992), "The sociology of scientific disciplines: on the genesis and stability of the disciplinary structure of modern science". Science in Context, 1 (5): 3-15.

THE WORLD bank \& the United nations. (2010), Natural hazards, unnatural disasters: the economics of effective prevention. Washington, D.C., The World Bank.

Tierney, K. J. (1994), “Aspectos socioeconómicos de la mitigación del peligro”. In: Lavell, A. (comp.). Alnorte del rio Grande - ciencias sociales, desastres: una perspectiva norteamericana. Bogota, La Red, pp. 93-112. 
. (2006), “Social inequality, hazards and disasters". In: Daniels, R. et al. Risk \& disasters: lessons from Hurricane Katrina. Philadelphia, University of Pennsylvania Press, pp. 110-125. . (2007), "From the margins to the mainstream? Disaster research at the crossroads". Annual Review Sociological, 33: 503-525.

Toвin, G. A. \& Montz, B. E. (1997), Natural hazards: explanation and integration. Nova York, The Guildford Press.

Trainor, J. E. \& Subbio, T. (2014), Critical issues in disaster science and management: a dialogue between researchers and practitioners. Washington, Fema.

Turner, Barry A. (1978), Man-made disasters. Nova York, Wykehan Publications.

UNISDR. (2015), The Pocket GAR 2015 - making development sustainable: the future of disaster risk management. Geneva, United Nations Office for Disaster Risk Reduction (UNISDR).

VALÊNCIO, N. et al. (2009), Sociologia dos desastres: construção, interfaces eperspectivas no Brasil. São Carlos, RiMa.

WALLACE, A. F. C. (1956), Human behavior in extreme situations: a survey of the literature and suggestions for further research. Washington, Disaster Study n. 1, National Academy of Science, National Research Council, publication 390.

WAUGH JR, W. L. (2000), Living with hazards, dealing with disasters: an introduction to emergency management. Armonk, M. E. Sharpe.

Wenger, D. (1978) “Community response to disaster," In: Quaranteli, E. (ed.), Disasters: theory and research. Bervely Hills, Sage.

Wевв, G. (1999), Individual and organizational response to natural disasters and other crisis events: the continuing value of the DRC Typology. Newark, Preliminary Paper 277, University of Delaware/Disaster Research.

Whitley, R. (2000), The intellectual and social organization of the science. Oxford, Oxford University Press.

Wisner, B. et al. (1994), At risk: natural hazards, people's vulnerability and disasters. Londres, Routledge.

\section{Resumo}

Dilemas e perspectivas da abordagem sociológica dos desastres naturais

O texto examina as controvérsias da abordagem sociológica dos desastres valendo-se dos subsídios extraídos da sociologia da ciência. Mais precisamente, considera os impasses cognitivos e políticos inerentes à sociologia dos desastres por meio das contribuições produzidas pelo estudo das controvérsias científicas. Sustenta que o desenvolvimento da abordagem sociológica dos desastres estrutura-se em torno de três controvérsias: (a) interdisciplinar: oposição evento-impacto; (b) intradisciplinar: oposição consenso-conflito; (c) extradisciplinar: oposição leigo-perito. Para tentar ultrapassar esses obstáculos, o texto apresenta o modelo Problema-Solução extraído do 
desenvolvimento da sociologia da ciência e da tecnologia. Com base nesse modelo teórico visa apresentar uma nova definição de desastres. Para desenvolver esse argumento, o texto foi dividido em cinco partes principais: (1) inicia com uma apresentação da problemática sociotécnica do estudo dos desastres; (2) segue com a apresentação das controvérsias interdisciplinares; (3) em seguida, das controvérsias intradisciplinares; (4) e das controvérsias extradisciplinares; (5) e termina com a formulação de uma nova definição de desastre.

Palavras-chave: Desastres naturais; Sociologia; Controvérsias; Sociotécnica.

\section{Abstract}

\section{Dilemmas and perspectives of the sociological approach on natural disasters}

The text examines the controversies of the sociological approach on disasters using the subsidies extracted from the sociology of science. More precisely, it considers the cognitive and political impasses inherent in the sociology of disasters through the contributions produced by the study of scientific controversies. He argues that the development of the sociological approach on disasters is structured around three controversies: (a) interdisciplinary: event-impact opposition; (b) intradisciplinary: consensus-conflict opposition; (c) extradisciplinary: lay-expert opposition. In order to overcome these obstacles, the text presents the model Problem-Solution extracted from the development on the sociology of science and technology. Based on this theoretical model aims to present a new definition of disasters. In order to develop this argument, the text was divided into five main parts: (1) it begins with a presentation of the socio-technical problems on the study of disasters; (2) follows with the presentation about the interdisciplinary controversies; (3) thereafter, the intradisciplinary controversies; (4) the extradisciplinary disputes; (5) and it ends with the formulation of a new definition of disaster.

Keywords: Natural disasters; Sociology; Controversies; Sociotechnical.

Texto recebido em 2/3/2017 e aprovado em 1/7/2017. DoI: 10.11606/0103-2070. ts.2017.111685.

marcos antonio mattedi é professor e coordenador do Programa de Pós-graduação em Desenvolvimento Regional da Universidade Regional de Blumenau. Dirige o Grupo de Pesquisa Núcleo de Estudos da Tecnociência (NET). E-mail: mattediblu@gmail.com. 